\title{
An Investigation on LTE Mobility Management
}

\author{
Ren-Huang Liou, Yi-Bing Lin, Fellow, IEEE, and Shang-Chih Tsai \\ Department of Computer Science \\ National Chiao Tung University \\ $\{$ rhliou, liny, tsaisc\}@cs.nctu.edu.tw
}

\begin{abstract}
Mobility management in Long Term Evolution (LTE) is different from that in the third generation mobile telecom networks. In LTE, the Mobility Management Entity (MME) is responsible for the mobility management function. The MME is connected to a large number of evolved Node Bs (cells) that are grouped into the Tracking Areas (TAs). The TAs are further grouped into TA Lists (TALs). When a User Equipment (UE) moves out of the current TAL, it reports its new location to the MME. If the LTE network attempts to connect to the UE, the MME asks the cells in the TAL to page the UE. In LTE paging, the MME may sequentially page a cell, the TA of the cell, and/or TAL of the cell. This paper investigates the performance of LTE paging, and provides the guidelines for the best paging sequence of cells.
\end{abstract}

Index Terms: Location update, Long Term Evolution (LTE), mobility management, paging.

\section{Introduction}

In a mobile telecom network, the locations of the User Equipments (UEs) are tracked so that incoming calls can be delivered to the UEs. Typical mobility management procedures include location update and paging. When a UE moves from one location to another location, the UE reports its new location to the network through the location update procedure. When 


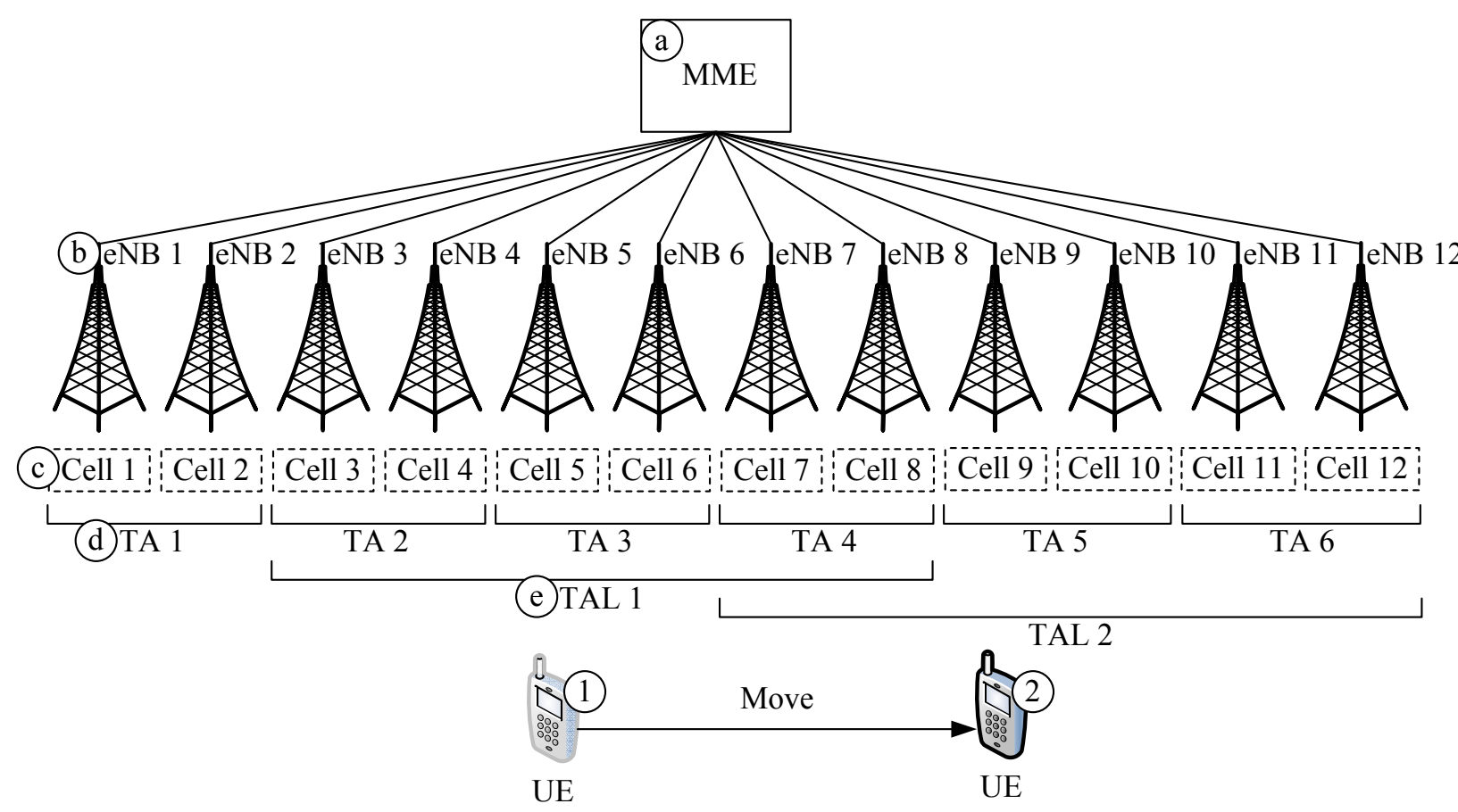

Figure 1: LTE Mobility Management Architecture

an incoming call to the UE arrives, the network identifies the location of the UE via the paging procedure.

In Long Term Evolution (LTE), the Mobility Management Entity (MME; Figure 1 (a)) is responsible for the mobility management function [1, 2], which is connected to a group of evolved Node Bs (eNBs; the LTE term for base stations; see Figure 1 (b)). The radio coverage of an eNB (or a sector of the eNB) is called a cell (see the dashed squares; Figure 1 (c)). Every cell has a unique cell identity. The cells are grouped into the Tracking Areas (TAs; e.g., TA 1 contains Cell 1 and Cell 2 in Figure 1 (d)). Every TA has a unique TA identity (TAI). The TAs are further grouped into TA Lists (TALs) [1]. In Figure 1, TAL 1 consists of TA 2, TA 3 and TA 4 (Figure 1 (e)).

A UE stores the TAL that includes the TA where the UE resides. In Figure 1 (1), the UE is covered by Cell 5 , and the TAL it stores is TAL $1=\{\mathrm{TA} 2$, TA 3 , TA 4$\}$. If the LTE network attempts to connect to the UE, it asks the cells in the TAL (e.g., Cell 3-Cell 8) to page the UE. Every eNB periodically broadcasts its TAI. The UE listens to the broadcast 
TAI and checks if the received TAI is in its TAL. If so, it means that the UE does not move out of the current location. In Figure 1, when the UE moves from Cell 5 to Cell 7, it receives the TA 4 identity broadcast from eNB 7 . Since TA 4 is included in TAL 1 , the UE still resides in the same location. When the UE moves to Cell 9 (Figure 1 (2)), the received TA 5 identity (broadcast from eNB 9) is not found in TAL 1, which means that the UE has moved out of the current location. In this case the UE executes the location update procedure to inform the MME that it has left TAL 1. The MME then assigns a new TAL to the UE. In Figure 1 , the new TAL is TAL $2=\{\mathrm{TA} 4$, TA 5 , TA 6$\}$. Note that the TAL is assigned on a per-user basis (i.e., TALs for different UEs may have different sizes and shapes), and the newly assigned TAL may be overlapped with the previously assigned TAL (e.g., TAL 2 is overlapped with TAL 1 in Figure 1). We consider the central policy [1] that assigns a new TAL whose central TA includes the cell where the UE currently resides. In Figure 1 (2), the UE resides in TA 5 and TAL $2=\{\mathrm{TA} 4$, TA 5 , TA 6$\}$ is centered at TA 5 . In the central policy, the TALs may be overlapped. For example, TA 4 is included in both TAL 1 and TAL 2.

When an incoming call to the UE arrives, it may incur large paging traffic if all cells in the TAL page the UE simultaneously. To resolve this issue, we implement three paging schemes in LTE. In this paper, an "interacted cell" refers to a cell where the UE is paged, makes calls, or performs location update. In other words, the interacted cell is the cell through which the UE had the interaction with the network.

Scheme CT (Cell-TAL). When an incoming call arrives, the MME first asks the last interacted cell to page the UE. If fails, all cells in the TAL are asked to page the UE.

Scheme TT (TA-TAL). When an incoming call arrives, the TA of the last interacted cell is asked to page the UE. If fails, all cells in the TAL are asked to page the UE.

Scheme CTT (Cell-TA-TAL). When an incoming call arrives, the MME first asks the 
last interacted cell to page the UE. If fails, the TA of the last interacted cell is asked to page the UE. If fails again, all cells in the TAL are asked to page the UE.

Basically, the central policy and the three paging schemes we described for LTE mobility management partially implement the movement-based and the distance-based location updates [3]-[5] with the Shortest-Distance-First (SDF) paging [4, 6]. Although these schemes have been intensively studied in the literature, they have not been exercised in any commercial mobile telecom network because their implementations are not feasible. Specifically, in the distance-based location update, the UE is required to have the cell topology information (i.e., the distance relationship between cells) $[3,7,8]$, which can not be practically implemented in a real network. In the SDF paging, it is difficult to dynamically define the neighboring cells (when the radio coverage changes, the "adjacent cells" may also change). Through the pre-defined TA configuration, LTE can partially implement the distance-based scheme with the SDF paging for commercial operation. In this paper, we show that LTE mobility management outperforms third-generation (3G) mobility management by capturing the advantages of the distance-based scheme with the SDF paging. We propose an analytic model to evaluate the performance of the TAL-based location update with the above three paging schemes.

This paper is organized as follows. Section 2 introduces the TAL-based location update. Section 3 proposes an analytic model for modeling the TAL-based scheme. Section 4 investigates the performance of the TAL-based scheme by numerical examples, and the conclusions are given in Section 5.

\section{TAL-based Location Update}

This section describes the TAL-based location update by considering one-dimensional (1-D) random walk model for the UE movement. This configuration significantly simplifies 


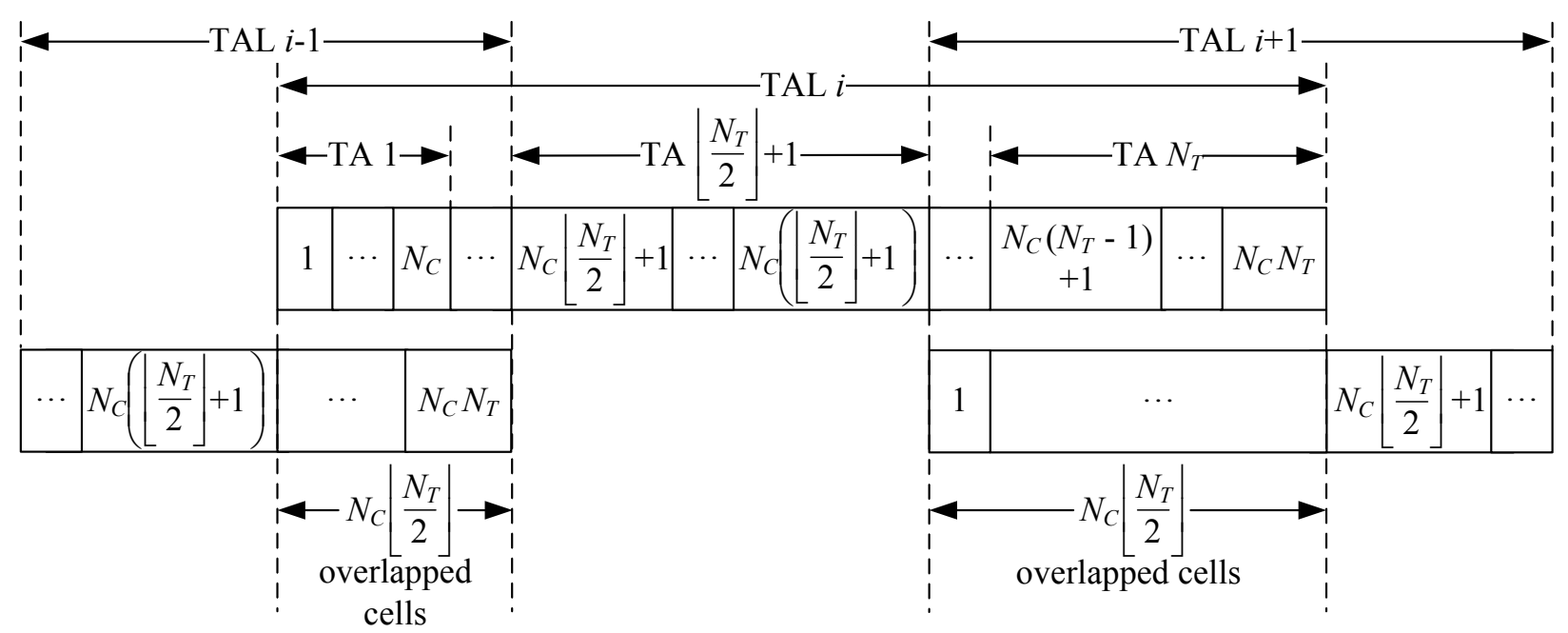

Figure 2: The TAL Configuration

the description and provides better demonstration. Also, 1-D configuration does exist in real environment [9]. We will extend the 1-D model to a 2-D model through simulation.

Figure 2 illustrates the TAL configuration where a rectangular represents a cell. In this configuration, a TAL contains $N_{T}$ TAs and each TA covers $N_{C}$ cells. In a TAL, the TAs are sequentially labeled from 1 to $N_{T}$, and the cells are sequentially labeled from 1 to $N_{C} N_{T}$. To simplify our discussion on the central policy mentioned in Section 1, we assume that $N_{T}$ is an odd number. Following the central policy, the TAL is overlapped with each of its adjacent TALs by $N_{C}\left\lfloor N_{T} / 2\right\rfloor$ cells. Therefore, when the UE leaves the current TAL from Cell $N_{C} N_{T}$, the entrance cell of the newly assigned TAL is Cell $N_{C}\left\lfloor N_{T} / 2\right\rfloor+1$. Similarly, if the UE leaves the current TAL from Cell 1, the entrance cell of the newly assigned TAL is Cell $N_{C}\left(\left\lfloor N_{T} / 2\right\rfloor+1\right)$.

In most commercial 3G mobile telecom networks, the base stations are grouped into Location Areas (LAs) [10]. When the UE moves from old LA to new LA, a location update is performed. When an incoming call arrives, all cells in the LA of the UE will page the UE. The 3G mobility management scheme is a special case of the TAL-based location update with the TT paging where the size of an LA is the size of a TAL, and $N_{T}=1$. 


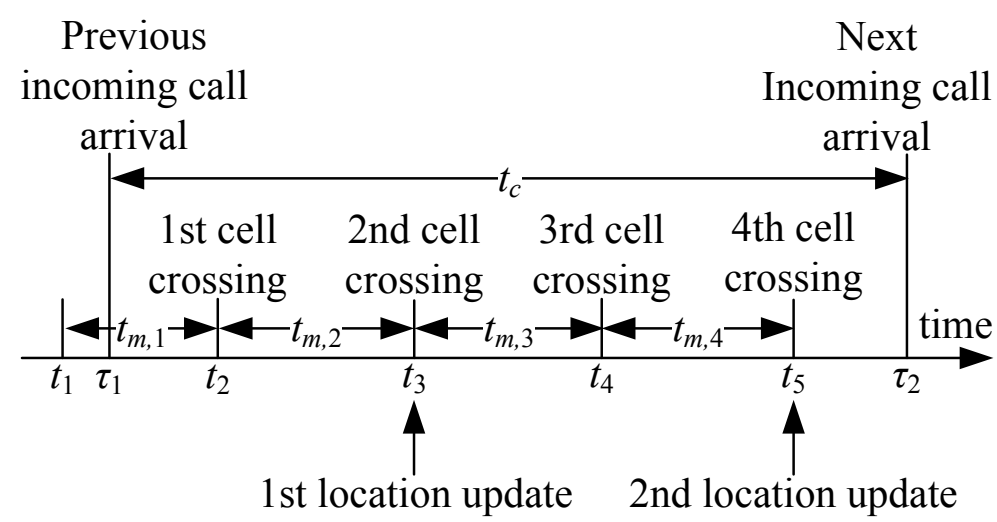

Figure 3: Timing Diagram for the Cell Crossings and the Incoming Call Arrivals

\section{Analytic Modeling}

This section models the TAL-based location update and the paging schemes. We first describe the input parameters and output measures.

Figure 3 illustrates the timing diagram for the cell crossings and the incoming call arrivals. In this figure, we assume that the inter-call arrival interval $t_{c}=\tau_{2}-\tau_{1}$ is an exponential random variable with the mean $1 / \lambda_{c}$. Let $t_{m, i}=t_{i+1}-t_{i}$ be the cell residence time between the $(i-1)$ th cell crossing and the $i$ th cell crossing. Assume that $t_{m, i}$ is independent and identically distributed random variable with the mean $1 / \lambda_{m}$, the variance $V$, and the Laplace transform $f_{m}^{*}(s)$. When the UE makes cell crossing, the UE moves to the right-hand side neighboring cell with routing probability $p$, and moves to the left-hand side neighboring cell with probability $1-p$. We consider the following three output measures:

- $C_{u}$ : the expected number of location updates during $t_{c}$

- $C_{p, x}$ : the expected number of cells that page the UE when an incoming call arrives, where $x \in\{C T, T T, C T T\}$

- $C_{d, x}$ : the expected number of polling cycles [6] before the UE is found, where $x \in$ $\{C T, T T, C T T\}$. Note that the maximum number of polling cycles for the CT, the TT, and the CTT schemes are 2, 2, and 3, respectively. 
It is clear that the smaller the above output measures, the better the performance of the location update and paging schemes.

We first derive $C_{u}$. Let $E[M]$ be the expected number of cell crossings before the UE leaves the current TAL (i.e., the expected number of cell crossings between two consecutive location updates). Then $C_{u}$ can be computed as

$$
C_{u}=\frac{E\left[t_{c}\right]}{E[M] E\left[t_{m, i}\right]}=\frac{\lambda_{m}}{\lambda_{c} E[M]}
$$

In [11], we have derived $E[M]$ with the following final formats. If $p=0.5$,

$$
E[M]=N_{C}\left(\left\lfloor\frac{N_{T}}{2}\right\rfloor+1\right)\left(N_{C}\left\lfloor\frac{N_{T}}{2}\right\rfloor+1\right)
$$

If $p \neq 0.5$,

$$
\begin{aligned}
E[M]= & {\left[\frac{A}{1+A-B}\right]\left[\frac{\left(N_{C} N_{T}+1\right) B-N_{C}\left\lfloor\frac{N_{T}}{2}\right\rfloor-1}{2 p-1}\right] } \\
& +\left[\frac{1-B}{1+A-B}\right]\left[\frac{\left(N_{C} N_{T}+1\right) A-N_{C}\left(\left\lfloor\frac{N_{T}}{2}\right\rfloor+1\right)}{2 p-1}\right]
\end{aligned}
$$

where

$$
A=\frac{1-\left(\frac{1-p}{p}\right)^{N_{C}\left(\left\lfloor\frac{N_{T}}{2}\right\rfloor+1\right)}}{1-\left(\frac{1-p}{p}\right)^{N_{C} N_{T}+1}} \text { and } B=\frac{1-\left(\frac{1-p}{p}\right)^{N_{C}\left\lfloor\frac{N_{T}}{2}\right\rfloor+1}}{1-\left(\frac{1-p}{p}\right)^{N_{C} N_{T}+1}}
$$

Now we derive $C_{p, C T}, C_{p, T T}$, and $C_{p, C T T}$. Figure 4 illustrates the state-transition diagram for the TAL-based location update. In this figure, state $j$ represents that the UE resides in Cell $j$ of the TAL, where $1 \leq j \leq N_{C} N_{T}$. For $2 \leq j \leq N_{C} N_{T}-1$, the UE moves from state $j$ to state $j+1$ with probability $p$, and moves from state $j$ to state $j-1$ with probability $1-p$. The state transition from state 1 to state $N_{C}\left(\left\lfloor N_{T} / 2\right\rfloor+1\right)$ represents that the UE 


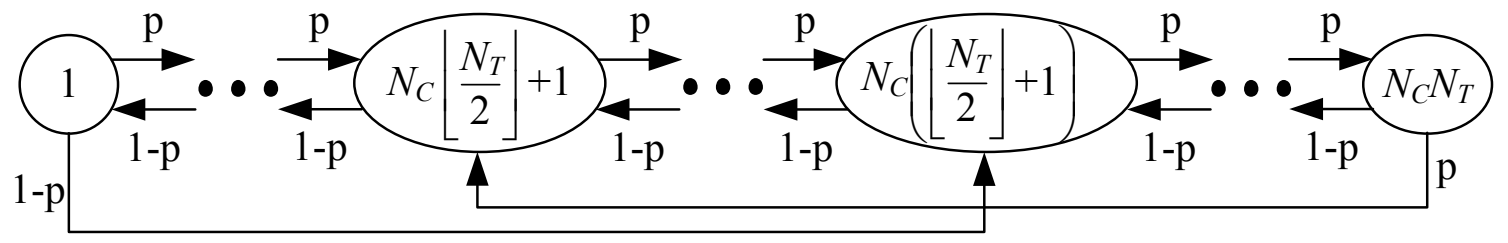

Figure 4: State-Transition Diagram for TAL-based Location Update

leaves the current TAL from Cell 1 and enters Cell $N_{C}\left(\left\lfloor N_{T} / 2\right\rfloor+1\right)$ of the newly assigned TAL. Similarly, the transition from state $N_{C} N_{T}$ to state $N_{C}\left\lfloor N_{T} / 2\right\rfloor+1$ represents that the UE leaves the current TAL from Cell $N_{C} N_{T}$ and enters Cell $N_{C}\left\lfloor N_{T} / 2\right\rfloor+1$ of the newly assigned TAL. Let $\pi_{i}$ be the steady-state probability that the UE resides in Cell $i$. From Figure 4, we obtain the following balance equations:

$$
\pi_{i}= \begin{cases}(1-p) \pi_{i+1}, & \text { for } i=1 \\ p \pi_{i-1}+(1-p) \pi_{i+1}, & \text { for } 2 \leq i \leq N_{C}\left\lfloor\frac{N_{T}}{2}\right\rfloor, \\ & N_{C}\left\lfloor\frac{N_{T}}{2}\right\rfloor+2 \leq i \leq N_{C}\left(\left\lfloor\frac{N_{T}}{2}\right\rfloor+1\right)-1 \\ & \text { and } N_{C}\left(\left\lfloor\frac{N_{T}}{2}\right\rfloor+1\right)+1 \leq i \leq N_{C} N_{T}-1 \\ p \pi_{i-1}+(1-p) \pi_{i+1}+p \pi_{N_{C} N_{T}}, & \text { for } i=N_{C}\left\lfloor\frac{N_{T}}{2}\right\rfloor+1 \\ p \pi_{i-1}+(1-p) \pi_{i+1}+(1-p) \pi_{1}, & \text { for } i=N_{C}\left(\left\lfloor\frac{N_{T}}{2}\right\rfloor+1\right) \\ p \pi_{i-1}, & \text { for } i=N_{C} N_{T}\end{cases}
$$

By rearranging (5), if $p=0.5, \pi_{i}$ can be re-written as

$$
\pi_{i}= \begin{cases}i \pi_{1}, & \text { for } 1 \leq i \leq N_{c}\left\lfloor\frac{N_{T}}{2}\right\rfloor+1 \\ \left(N_{C}\left\lfloor\frac{N_{T}}{2}\right\rfloor+1\right) \pi_{1}, & \text { for } N_{c}\left\lfloor\frac{N_{T}}{2}\right\rfloor+1<i \leq N_{c}\left(\left\lfloor\frac{N_{T}}{2}\right\rfloor+1\right) \\ \left(N_{C} N_{T}-i+1\right) \pi_{1}, & \text { for } N_{c}\left(\left\lfloor\frac{N_{T}}{2}\right\rfloor+1\right)<i \leq N_{C} N_{T}\end{cases}
$$

If $p \neq 0.5$ and $N_{T}=1, \pi_{i}$ is re-written as

$$
\pi_{i}=\pi_{1}, \text { for } 1 \leq i \leq N_{C}
$$


If $p \neq 0.5$ and $N_{T} \neq 1, \pi_{i}$ is re-written as

$$
\pi_{i}=\left\{\begin{array}{lc}
\left(\frac{1}{1-p}\right)\left\{1+\left(\frac{p^{2}}{2 p-1}\right)\left[\left(\frac{p}{1-p}\right)^{i-2}-1\right]\right\} \pi_{1}, & \text { for } 2 \leq i \leq N_{C}\left\lfloor\frac{N_{T}}{2}\right\rfloor+1 \\
\left(\frac{1}{1-p}\right)\left\{1+\left(\frac{p^{2}}{2 p-1}\right)\left[\left(\frac{p}{1-p}\right)^{i-2}-1\right]\right\} \pi_{1} & \\
-\left[\left(\frac{p}{1-p}\right)^{i-N_{C}\left\lfloor\frac{N_{T}}{2}\right\rfloor-1}-1\right]\left(\frac{p}{2 p-1}\right) \pi_{N_{C} N_{T},} & \text { for } N_{C}\left\lfloor\frac{N_{T}}{2}\right\rfloor+1<i \\
\left\{1+\left[\frac{(1-p)^{2}}{1-2 p}\right]\left[\left(\frac{1-p}{p}\right)^{N_{C} N_{T}-i-1}-1\right]\right\} & \leq N_{C}\left(\left\lfloor\frac{N_{T}}{2}\right\rfloor+1\right) \\
\times\left(\frac{\left.\pi_{N_{C} N_{T}}^{p}\right),}{p}\right) & \text { for } N_{C}\left(\left\lfloor\frac{N_{T}}{2}\right\rfloor+1\right) \leq i
\end{array}\right.
$$

and

$$
\begin{aligned}
\pi_{N_{C} N_{T}}= & \left(\frac{1}{1-p}\right)\left\{1+\left(\frac{p^{2}}{2 p-1}\right)\left[\left(\frac{p}{1-p}\right)^{N_{C}\left\lfloor\frac{N_{T}}{2}\right\rfloor+N_{C}-2}-1\right]\right\}\left\{\left(\frac{p}{1-p}\right)^{N_{C}-1}-1\right] \\
& \left.\times\left(\frac{p}{2 p-1}\right)+\frac{1}{p}+\left[\frac{(1-p)^{2}}{p(1-2 p)}\right]\left[\left(\frac{1-p}{p}\right)^{N_{C}\left\lfloor\frac{N_{T}}{2}\right\rfloor-1}-1\right]\right\}^{-1} \pi_{1}
\end{aligned}
$$

Probability $\pi_{i}$ can be solved by using $\sum_{i=1}^{N_{C} N_{T}} \pi_{i}=1$ and equations (6)-(9).

Let $q_{i, j}^{(k)}$ be the probability that the UE moves from Cell $i$ to Cell $j$ after $k$ cell crossings without any location update (it is possible that the Cell $j$ is revisited several times during these $k$ cell crossings). Let $\bar{q}_{i, 0}^{(k)}$ and $\bar{q}_{i, N_{C} N_{T}+1}^{(k)}$ be the probabilities that the UE initially stays in Cell $i$ and moves out of the current TAL from Cell 1 and Cell $N_{C} N_{T}$ at the $k$ th cell crossing, respectively. Probabilities $q_{i, j}^{(k)}, \bar{q}_{i, 0}^{(k)}$, and $\bar{q}_{i, N_{C} N_{T}+1}^{(k)}$ are derived as follows. To compute the probability that the UE leaves the current TAL at the $k$ th cell crossing (i.e., $\bar{q}_{i, 0}^{(k)}$ and $\bar{q}_{i, N_{C} N_{T}+1}^{(k)}$ ), Figure 5 modifies Figure 4 by adding two absorbing states 0 and $N_{C} N_{T}+1$ 


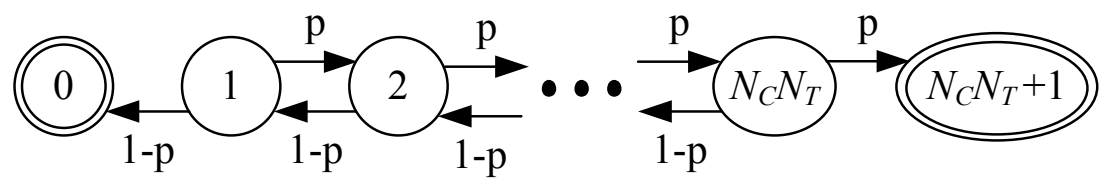

Figure 5: State-Transition Diagram for 1-D Random Walk Model with Two Absorbing States and removing the transitions from state 1 to state $N_{C}\left(\left\lfloor N_{T} / 2\right\rfloor+1\right)$ and from $N_{C} N_{T}$ to $N_{C}\left\lfloor N_{T} / 2\right\rfloor+1$. Let $q_{i, j}$ be the one-step transition probability from state $i$ to state $j$. Figure 5 illustrates the state-transition diagram, where the transition probability matrix $Q=\left(q_{i, j}\right)$ of the random walk is

$$
Q=\left(\begin{array}{cccccccc}
1 & 0 & 0 & 0 & \cdots & 0 & 0 & 0 \\
1-p & 0 & p & 0 & \cdots & 0 & 0 & 0 \\
0 & 1-p & 0 & p & \cdots & 0 & 0 & 0 \\
\vdots & \vdots & \vdots & \vdots & \ddots & \vdots & \vdots & \vdots \\
0 & 0 & 0 & 0 & \cdots & 0 & p & 0 \\
0 & 0 & 0 & 0 & \cdots & 1-p & 0 & p \\
0 & 0 & 0 & 0 & \cdots & 0 & 0 & 1
\end{array}\right)_{\left(N_{C} N_{T}+2\right) \times\left(N_{C} N_{T}+2\right)}
$$

Let $Q^{(k)}=\left(q_{i, j}^{(k)}\right)$ be the transition matrix. From $(10), Q^{(k)}$ is computed by the matrix multiplication $Q^{(k)}=\prod_{i=1}^{k} Q$. Based on $q_{i, j}^{(k)}, \bar{q}_{i, 0}^{(k)}$ and $\bar{q}_{i, N_{C} N_{T}+1}^{(k)}$ are computed as

$$
\bar{q}_{i, 0}^{(k)}= \begin{cases}q_{i, 0}, & \text { for } k=1 \\ q_{i, 0}^{(k)}-q_{i, 0}^{(k-1)}, & \text { for } k>1\end{cases}
$$

and

$$
\bar{q}_{i, N_{C} N_{T}+1}^{(k)}= \begin{cases}q_{i, N_{C} N_{T}+1}, & \text { for } k=1 \\ q_{i, N_{C} N_{T}+1}^{(k)}-q_{i, N_{C} N_{T}+1}^{(k-1)}, & \text { for } k>1\end{cases}
$$


Given that the UE resides in Cell $i$ when the previous incoming call arrives (i.e., Cell $i$ is an "interacted cell"; see $\tau_{1}$ in Figure 3$)$, let $\phi_{i}^{(k, n)}$ be the probability that after $k$ cell crossings and $n$ location updates, the UE moves back to the last interacted cell. If $n=0$, then the last interacted cell is Cell $i$. If $n \geq 1$, the last interacted cell is the cell where the UE performs the $n$th location update. From (11) and $(12), \phi_{i}^{(k, n)}$ is computed as

$$
\phi_{i}^{(k, n)}= \begin{cases}1, & \text { for } k=0 \text { and } n=0 \\ q_{i, i}^{(k)}, & \text { for } k>0 \text { and } n=0 \\ \sum_{j=1}^{k}\left\{\phi_{N_{C}\left[\frac{N_{T}}{2}\right\rfloor+1}^{(k-j, n-1)} \bar{q}_{i, N_{C} N_{T}+1}^{(j)}+\phi_{N_{C}\left(\left\lfloor\frac{N_{T}}{2}\right\rfloor+1\right)}^{(k-j, n-1)} \bar{q}_{i, 0}^{(j)}\right\} & \text { for } k \geq n \geq 1 \\ 0, & \text { for } k<n\end{cases}
$$

In (13), for $k=0$ and $n=0$, the UE does not make any cell crossing (i.e., the UE still stays in Cell $i$ ), and $\phi_{i}^{(0,0)}=1$. For $k>0$ and $n=0$, the last interacted cell is Cell $i$, and the probability that the UE moves from Cell $i$ back to Cell $i$ after $k$ cell crossings without any location update is $q_{i, i}^{(k)}$. For $k \geq n \geq 1$, two cases are considered. First, if the UE moves out of the current TAL from Cell $N_{C} N_{T}$ at the $j$ th cell crossing (with probability $\bar{q}_{i, N_{C} N_{T}+1}^{(j)}$ ), the last interacted cell is Cell $N_{C}\left\lfloor N_{T} / 2\right\rfloor+1$ of the newly assigned TAL, and the numbers of the remaining cell crossings and location updates are $k-j$ and $n-1$, respectively. Second, if the UE moves out of the current TAL from Cell 1 at the $j$ th cell crossing (with probability $\bar{q}_{i, 0}^{(j)}$ ), the last interacted cell is Cell $N_{C}\left(\left\lfloor N_{T} / 2\right\rfloor+1\right)$ of the newly assigned TAL, and the numbers of the remaining cell crossings and location updates are $k-j$ and $n-1$, respectively. For $k<n$, it is impossible that the number of cell crossings is smaller than that of location updates. Therefore, $\phi_{i}^{(k, n)}=0$ for $k<n$.

Let $\beta(k)$ be probability that the UE makes $k$ cell crossings during $t_{c}$. From our previous 
work $[6], \beta(k)$ is derived as

$$
\beta(k)= \begin{cases}1-\left(\frac{\lambda_{m}}{\lambda_{c}}\right)\left[1-f_{m}^{*}\left(\lambda_{c}\right)\right], & \text { for } k=0 \\ \left(\frac{\lambda_{m}}{\lambda_{c}}\right)\left[1-f_{m}^{*}\left(\lambda_{c}\right)\right]^{2}\left[f_{m}^{*}\left(\lambda_{c}\right)\right]^{k-1}, & \text { for } k>0\end{cases}
$$

Assume that $t_{m, i}$ is a Gamma random variable with the mean $1 / \lambda_{m}$, the variance $V$, and the Laplace transform

$$
f_{m}^{*}(s)=\left(\frac{1}{V \lambda_{m} s+1}\right)^{\frac{1}{V \lambda_{m}^{2}}}
$$

We consider the Gamma distribution because it has been shown that the distribution of any positive random variable can be approximated by a mixture of Gamma distributions [12]. The Gamma distribution was used to model UE movement in many studies [5, 6, 9] and is used in this paper to investigate the impact of variance for cell residence times. From (15), (14) is re-written as

$$
\beta(k)= \begin{cases}1-\left(\frac{\lambda_{m}}{\lambda_{c}}\right)\left[1-\left(\frac{1}{V \lambda_{m} \lambda_{c}+1}\right)^{\frac{1}{V \lambda_{m}^{2}}}\right], & \text { for } k=0 \\ \left(\frac{\lambda_{m}}{\lambda_{c}}\right)\left[1-\left(\frac{1}{V \lambda_{m} \lambda_{c}+1}\right)^{\frac{1}{V \lambda_{m}^{2}}}\right]^{2}\left(\frac{1}{V \lambda_{m} \lambda_{c}+1}\right)^{\frac{k-1}{V \lambda_{m}^{2}}}, & \text { for } k>0\end{cases}
$$

Let $\theta_{C}$ be the probability that the UE resides in the last interacted cell when an incoming call arrives. From (6)-(9), (13) and (16), $\theta_{C}$ is expressed as

$$
\theta_{C}=\sum_{k=0}^{\infty} \beta(k) \sum_{i=1}^{N_{C} N_{T}} \pi_{i} \sum_{n=0}^{k} \phi_{i}^{(k, n)}
$$

In the right-hand side of (17), the UE makes $k$ cell crossings during $t_{c}$ with probability $\beta(k)$ $(0 \leq k \leq \infty)$ and resides in Cell $i$ with probability $\pi_{i}\left(1 \leq i \leq N_{C} N_{T}\right)$ when the previous incoming call arrives. Therefore, $\theta_{C}$ is the summation of the product $\beta(k) \pi_{i} \phi_{i}^{(k, n)}$ over all 
possible $(k, i, n)$ pairs (i.e., $0 \leq n \leq k \leq \infty$ and $1 \leq i \leq N_{C} N_{T}$ ).

Similar to $\phi_{i}^{(k, n)}$, let $\eta_{i}^{(k, n)}$ be the probability that after $k$ cell crossings and $n$ location updates, the UE moves back to the TA of the last interacted cell given that the UE resides in Cell $i$ when the previous incoming call arrives. From (11) and (12), $\eta_{i}^{(k, n)}$ is derived as

$$
\eta_{i}^{(k, n)}= \begin{cases}1, \quad & \text { for } k=0 \text { and } n=0 \\ \sum_{j=N_{C}\left\lceil\frac{i}{N_{C}}\right\rceil-N_{C}+1}^{N_{C}\left\lceil\frac{i}{N_{C}}\right\rceil} q_{i, j}^{(k)}, & \text { for } k>0 \text { and } n=0 \\ \sum_{j=1}^{k}\left\{\eta_{N_{C}\left\lfloor\frac{N_{T}}{2}\right\rfloor+1}^{(k-j, n-1)} \bar{q}_{i, N_{C} N_{T}+1}^{(j)}+\eta_{N_{C}\left(\left\lfloor\frac{N_{T}}{2}\right\rfloor+1\right)}^{(k-j)} \bar{q}_{i, 0}^{(j)}\right\} & \text { for } k \geq n \geq 1 \\ 0, & \text { for } k<n\end{cases}
$$

The explanation of (18) is similar to that of (13), and the details are omitted.

Let $\theta_{T}$ be the probability that the UE resides in the TA of the last interacted cell when an incoming call arrives. Similar to (17), from (6)-(9), (16) and (18), $\theta_{T}$ is expressed as

$$
\theta_{T}=\sum_{k=0}^{\infty} \beta(k) \sum_{i=1}^{N_{C} N_{T}} \pi_{i} \sum_{n=0}^{k} \eta_{i}^{(k, n)}
$$

From (17) and (19), $C_{p, x}$ is computed as

$$
\begin{aligned}
& C_{p, C T}=\theta_{C}+\left(1-\theta_{C}\right)\left(1+N_{C} N_{T}\right) \\
& C_{p, T T}=\theta_{T} N_{C}+\left(1-\theta_{T}\right)\left(N_{C}+N_{C} N_{T}\right)
\end{aligned}
$$

and

$$
C_{p, C T T}= \begin{cases}C_{p, C T}, & \text { for } N_{C}=1 \\ \theta_{C}+\left(\theta_{T}-\theta_{C}\right)\left(1+N_{C}\right)+\left(1-\theta_{T}\right)\left(1+N_{C}+N_{C} N_{T}\right), & \text { for } N_{C} \neq 1\end{cases}
$$


Similar to the derivations for $C_{p, x}, C_{d, x}$ is computed as

$$
\begin{aligned}
& C_{d, C T}=\theta_{C}+2\left(1-\theta_{C}\right)=2-\theta_{C} \\
& C_{d, T T}=\theta_{T}+2\left(1-\theta_{T}\right)=2-\theta_{T}
\end{aligned}
$$

and

$$
C_{d, C T T}= \begin{cases}C_{d, C T}, & \text { for } N_{C}=1 \\ \theta_{C}+2\left(\theta_{T}-\theta_{C}\right)+3\left(1-\theta_{T}\right)=3-\theta_{C}-\theta_{T}, & \text { for } N_{C} \neq 1\end{cases}
$$

The major differences between our analytic model and the previous models for the movement-based and the distance-based schemes $[5,6]$ are described as follows.

- In the movement-based and the distance-based schemes, a location update or an incoming call resets the center cell of the residing area [6] to be the last interacted cell. On the other hand, the TAL-based location update resets the central TA of the TAL to be the TA of the last interacted cell (i.e., the last interacted cell may not be the center cell of the TAL). Therefore, our analytic model is more complicated than those for the movement-based and the distance-based location updates.

- In the SDF paging, the last interacted cell is the center cell of the subareas (i.e., the TA) [6]. In the $\mathrm{TT}$ and the CTT schemes, the last interacted cell may not be the center cell of the TA.

- The previous analytic models $[5,6]$ assume that the UE moves from a cell to one of the neighboring cells with the same probability. In our model, the UE can move to each of its neighboring cells with different probabilities.

Our analytic model is used to validate the discrete event simulation model. Simulation experiments show that the discrepancies between the analytic (i.e., equations (1), (20)-(25)) 
and simulation results are within 1\%. After the simulation of 1-D cell configuration has been validated by the analytic model, the validated simulation flowchart is extended to accommodate the 2-D mesh cell configuration (i.e., Manhattan-street layout).

\section{Numerical Examples}

This section investigates the performance of the TAL-based location update and paging schemes. We first point out that the performance results for the 1-D configuration are consistent with those for the 2-D configuration. For $x \in\{C T, T T, C T T\}$, Figure 6 (a) plots $C_{p, x}$ for the 1-D configuration, and Figure 6 (b) plots $C_{p, x}$ for the 2-D configuration. Figures 6 (c) and (d) plot $C_{u}$ and $C_{d, x}$ for both the 1-D and the 2-D configurations, respectively. In the 1-D configuration, we consider the TAL size $N_{C} N_{T}=45$ and $p=0.5$. In the 2-D mesh configuration, we consider a $45 \times 45$ cell structure, and the UE moves to one of the four neighboring cells with the same routing probabilities 0.25 . This figure shows that the trends of $C_{p, x}, C_{u}$ and $C_{d, x}$ are similar for both the 1-D and the 2-D models. Therefore, our observations on the 1-D model are also valid for the 2-D model. For other input parameter setups, same results are observed, and the details are omitted.

The remainder of this section shows the results for the 1-D configuration because several non-trivial observations can be easily explained through the analytic model of this simple configuration.

\subsection{Analysis of the $C_{u}$ Performance}

In the $1-\mathrm{D}$ configuration, it suffices to consider $0.5 \leq p \leq 1$ in our study. In practice, a small $p$ (e.g., $p=0.5$; solid lines in Figure 7) represents the movement of a pedestrian or a vehicle in local roads, which exhibits locality. A large $p$ (e.g., $p=0.85$; dashed lines in 


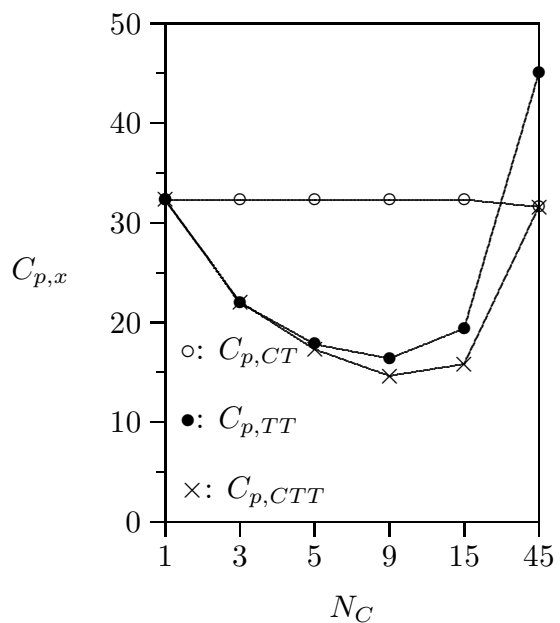

(a) $C_{p, x}$ for $1-\mathrm{D}$

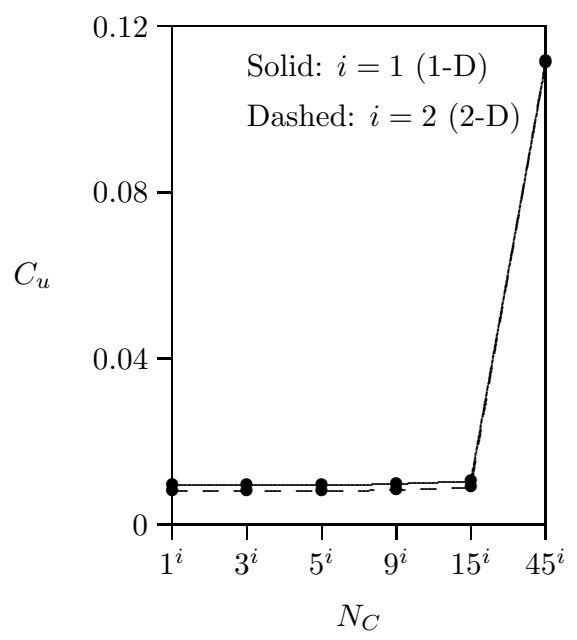

(c) $C_{u}$ for both 1-D and 2-D

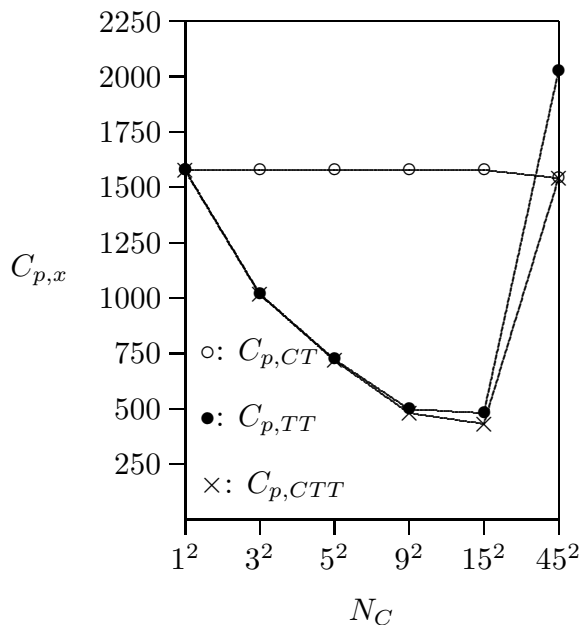

(b) $C_{p, x}$ for $2-\mathrm{D}$

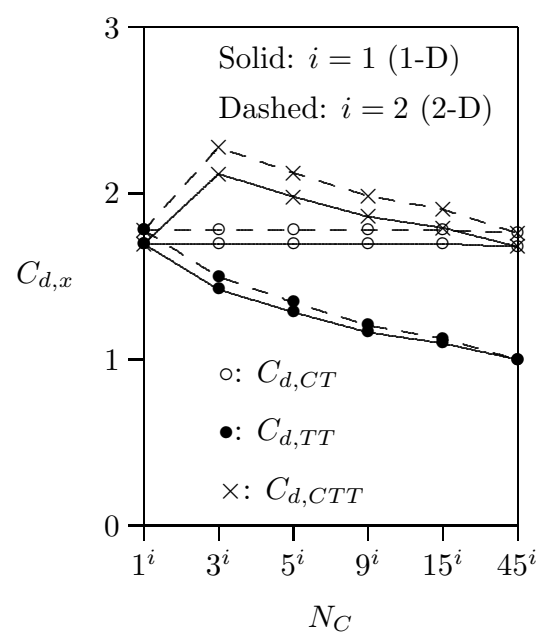

(d) $C_{d, x}$ for both 1 -D and 2-D

Figure 6: Performance Results for 1-D $\left(p=0.5\right.$ and $\left.N_{C} N_{T}=45\right)$ and 2-D Configurations $\left(p=0.25\right.$ and $\left.N_{C} N_{T}=45^{2}\right)$, where $\lambda_{m} / \lambda_{c}=5$ and $V=1 / \lambda_{m}^{2}$

Figure 7) represents the movement of a vehicle in highways. When $p$ increases, the UE tends to move to one direction. Therefore, $C_{u}$ increases as $p$ increases. In Figures 7 , the values of the dashed curves $(p=0.85)$ are higher than those of the solid curves $(p=0.5)$.

When $\lambda_{m} / \lambda_{c}$ increases (i.e., more cell crossings during $t_{c}$ ), more location updates are expected (i.e., $C_{u}$ increases as $\lambda_{m} / \lambda_{c}$ increases). In Figures 7 , the values of the $\bullet$ curves $\left(\lambda_{m} / \lambda_{c}=50\right)$ are higher than those of the $\circ$ curves $\left(\lambda_{m} / \lambda_{c}=5\right)$.

The effect of $N_{C}$ on $C_{u}$ can be explained as follows. When $N_{C}=1$, the entrance cell of 


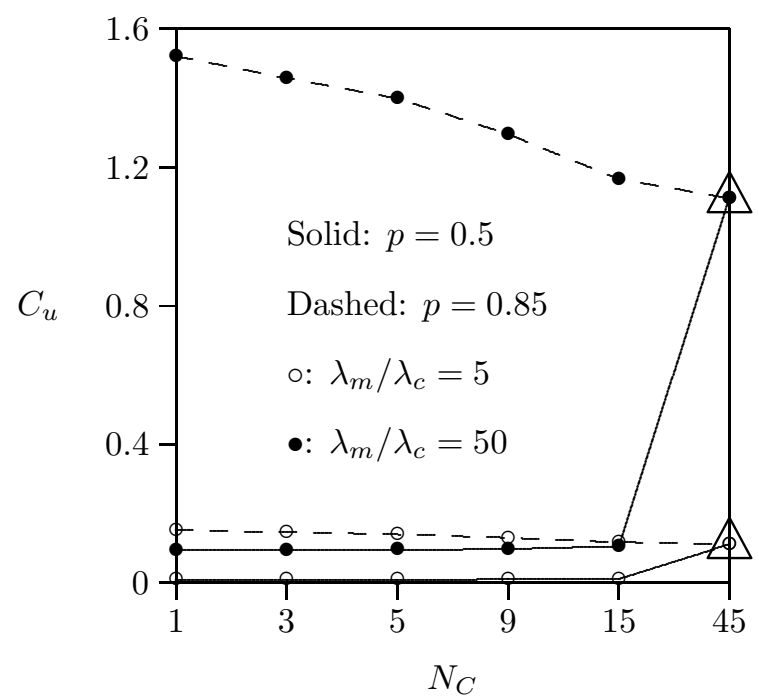

Figure 7: Effects of $p, \lambda_{m} / \lambda_{c}$, and $N_{C}$ on $C_{u}\left(N_{C} N_{T}=45\right)$

the UE is the center cell of the new TAL. On the other hand, when $N_{C}=45$ (i.e., $N_{T}=1$ or the TAL only has one TA), the entrance cell of the UE is the boundary cell of the new TAL. Now consider $p=0.5$. If $N_{C}=1$, then it is unlikely that the UE will move out of the TAL in first few cell crossings. On the other hand, if $N_{C}=45$, then the UE will move out of the TAL at the first cell crossing with probability 0.5. In other words, for a fixed $N_{C} N_{T}$ value, if $p$ is small, $E[M]$ decreases as $N_{C}$ increases. For example, for $N_{C} N_{T}=45$ and $p=0.5$, equation (2) indicates that $E[M]=529$ for $N_{C}=1$ and $E[M]=45$ for $N_{C}=45$. Since $C_{u}$ is inversely proportional to $E[M]$ (see (1)), for a fixed $N_{C} N_{T}$ value, $C_{u}$ increases as $N_{C}$ increases for a small $p$ (the solid curves in Figure 7).

On the other hand, for a fixed $N_{C} N_{T}$ value, if $p$ is large, $E[M]$ increases as $N_{C}$ increases. In the extreme case, when $p=1,(3)$ is re-written as $E[M]=N_{C}\left\lceil\frac{N_{T}}{2}\right\rceil$. If $N_{C} N_{T}=45$, we have $E[M]=23$ for $N_{C}=1$ and $E[M]=45$ for $N_{C}=45$. Therefore, for a large $p, C_{u}$ decreases as $N_{C}$ increases (the dashed curves in Figure 7).

As mentioned in Section 2, the 3G location update is a special case of the TAL-based scheme where $N_{T}=1$ (i.e., $N_{C}=45$; see the $\triangle$ symbol in Figure 7 ). When $p=0.5$, the TAL-based scheme with $N_{T} \neq 1$ can reduce about $90 \%$ of the $3 \mathrm{G}$ location update cost. 


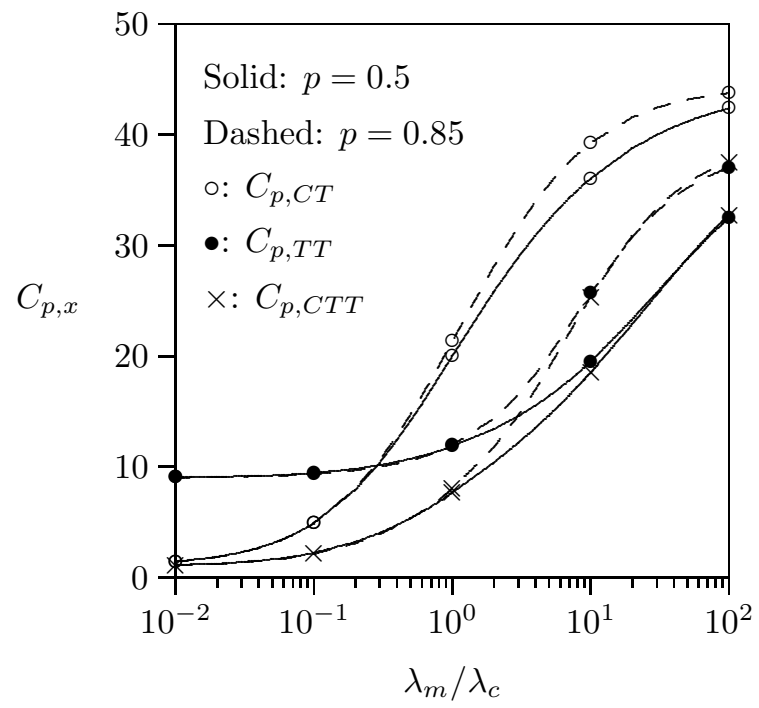

Figure 8: Effects of $\lambda_{m} / \lambda_{c}$ and $p$ on $C_{p, x}\left(N_{C}=9, N_{T}=5\right.$ and $\left.V=1 / \lambda_{m}^{2}\right)$

When $p=0.85$, the TAL-based scheme with $N_{T} \neq 1$ incurs extra $4 \%-36 \%$ cost over the $3 \mathrm{G}$ location update.

\subsection{Analysis of the $C_{p, x}$ Performance}

This subsection investigates the effects of $\lambda_{m} / \lambda_{c}, p, V$ and $N_{C}$ on $C_{p, x}$.

Effects of $\lambda_{m} / \lambda_{c}$ : For all $x \in\{C T, T T, C T T\}$, Figure 8 indicates that $C_{p, x}$ increase as $\lambda_{m} / \lambda_{c}$ increases. When $\lambda_{m} / \lambda_{c}$ increases, it is more likely that the UE is far away from the last interacted cell when an incoming call arrives, and thus higher $C_{p, x}$ are expected. When $\lambda_{m} / \lambda_{c}$ is small, the low-mobility UE is more likely to be found in the last interacted cell, and there is no need to page the TA. In this case, we observe that $C_{p, C T T}<C_{p, C T}<C_{p, T T}$. On the other hand, when $\lambda_{m} / \lambda_{c}$ is large, the UE is unlikely to reside in the last interacted cell, and paging the last interacted cell incurs extra paging cost. Therefore, $C_{p, C T T}$ is slightly larger than $C_{p, T T}$. The effect of $\lambda_{m} / \lambda_{c}$ on $C_{p, C T}$ is more significant than that on $C_{p, C T T}$, and than that on $C_{p, T T}$. In summary, for small $\lambda_{m} / \lambda_{c}, C_{p, C T T}<C_{p, C T}<C_{p, T T}$. For large $\lambda_{m} / \lambda_{c}, C_{p, C T T} \approx C_{p, T T}<C_{p, C T}$. 
Effects of $p$ : For all $x \in\{C T, T T, C T T\}$, Figure 8 indicates that a large $p$ incurs high paging costs $C_{p, x}$ because the UE may be far away from the last interacted cell when an incoming call arrives. The effect of $p$ becomes more significant when $\lambda_{m} / \lambda_{c}$ increases. Figure 9 shows that the effects of $p$ on $C_{p, x}$ become insignificant as $V$ increases. In Figures 8-10, for most $p$ values, $C_{p, C T T}$ are smaller than both $C_{p, C T}$ and $C_{p, T T}$.

Effects of $V$ : For a fixed $\lambda_{m}$ value, we have the following facts about the variance $V$ of the cell residence time $t_{m, i}$.

Fact 1. When $V$ is small (i.e., the user movement pattern is regular), most $t_{m, i}$ have values around $1 / \lambda_{m}$. In this case, if $\lambda_{m} / \lambda_{c}<1$, the UE is likely to be found in the last interacted cell when an incoming call arrives. On the other hand, if $\lambda_{m} / \lambda_{c}>1$, the UE is unlikely to be found in the last interacted cell.

Fact 2. For any $\lambda_{m}$ value, when $V$ increases (i.e,. the user movement pattern becomes irregular), more longer $t_{m, i}$ periods will be observed, and the UE does not move in many consecutive $t_{c}$ periods that fall in these $t_{m, i}$. In this case, the UE is always found in the last interacted cell, and lower $C_{p, x}$ are observed for all $x \in$ $\{C T, T T, C T T\}$

Due to Fact 1, when $V$ is small and $\lambda_{m} / \lambda_{c}>1$, the UE is unlikely to be found in the last interacted cell, and $C_{p, C T}>C_{p, T T}$ (in Figure 9 (a), for $V \leq 1 / \lambda_{m}^{2}$, the values of the o curves are higher than those of the $\bullet$ curves). On the other hand, $C_{p, C T}<C_{p, T T}$ for a small $\lambda_{m} / \lambda_{c}$ (in Figure $9(\mathrm{~b})$, for $V \leq 1 / \lambda_{m}^{2}$, the values of the $\circ$ curves are lower than those of the - curves). Due to Fact 2, Figure 9 shows that when $V$ is large, low $C_{p, x}$ are observed for all $x \in\{C T, T T, C T T\}$, and $C_{p, C T}<C_{p, T T}$. Specifically, when $V>30 / \lambda_{m}^{2}$, the values of the $\circ$ curves are lower than those of the $\bullet$ curves in Figure 9 (a), and the values of the o curves are lower than those of the $\bullet$ curves for all $V$ values in Figure 9 (b). The CTT scheme is a combination of the CT and the TT schemes, which explores the advantages of these two schemes in both large and 


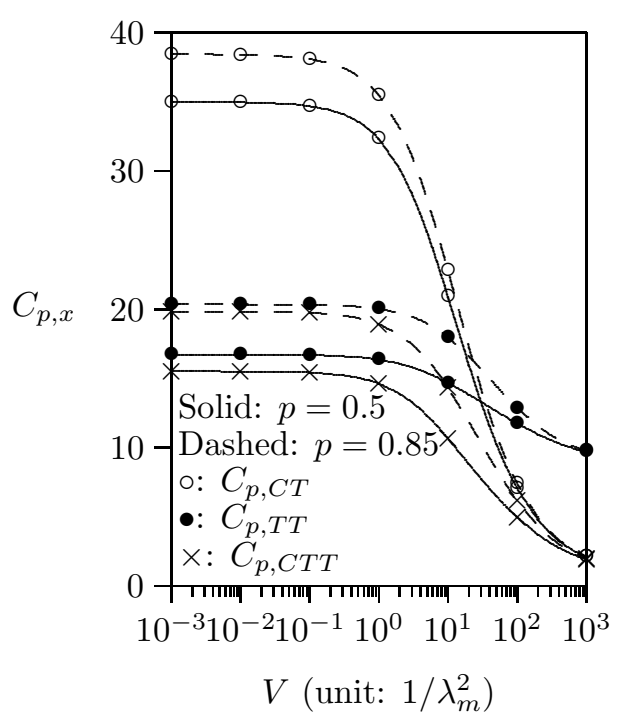

(a) $\lambda_{m} / \lambda_{c}=5$

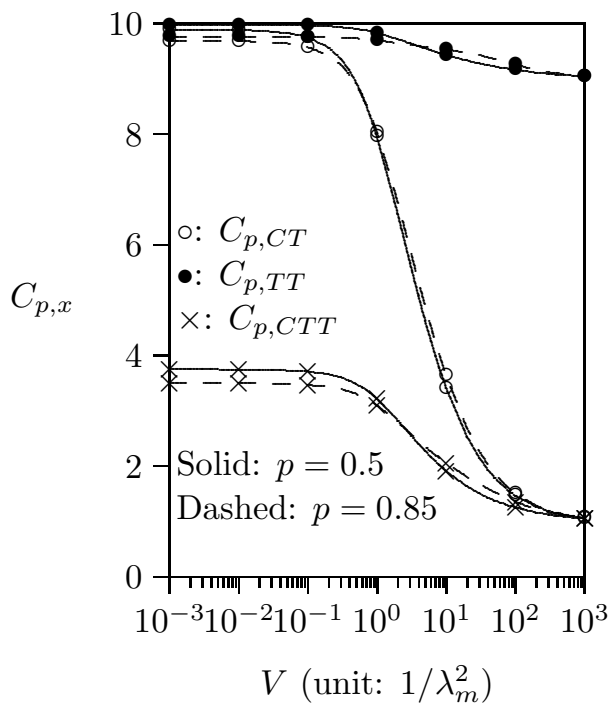

(b) $\lambda_{m} / \lambda_{c}=0.2$

Figure 9: Effects of $V$ and $p$ on $C_{p, x}\left(N_{C}=9\right.$ and $\left.N_{T}=5\right)$

small $V$ scenarios, and yields the best performance. The effect of $V$ on $C_{p, C T}$ is more significant than that on $C_{p, C T T}$, and than that on $C_{p, T T}$. In summary, when $V$ is small, $C_{p, C T T}<C_{p, C T}<C_{p, T T}$ for a small $\lambda_{m} / \lambda_{c}$, and $C_{p, C T T} \approx C_{p, T T}<C_{p, C T}$ for a large $\lambda_{m} / \lambda_{c}$. When $V$ is large, $C_{p, C T T}<C_{p, C T}<C_{p, T T}$.

Effects of $N_{C}$ : Since the CT scheme does not page the TA of the last interacted cell, $C_{p, C T}$ is not sensitive to the change of $N_{C}$ (see the o curves in Figure 10). Figure 10 (a) indicates that when $V$ is small and $\lambda_{m} / \lambda_{c}>1, C_{p, T T}$ decreases and then increases as $N_{C}$ increases. In this case, the UE is unlikely to reside in the last interacted cell (Fact 1), and a large $N_{C}$ results in a higher probability that the UE is found in the TA of the last interacted cell. Therefore, there is no need to page all cells in the TAL; i.e., lower $C_{p, T T}$ is observed. However, if $N_{C}$ is "too" large, the cost of paging the TA is high, and the benefit of paging the TA becomes insignificant. Therefore, $C_{p, T T}$ decreases and then increases as $N_{C}$ increases. Figure 10 (b) indicates that when $V$ is large and $\lambda_{m} / \lambda_{c}<1, C_{p, T T}$ increases as $N_{C}$ increases. In this case, the UE is more likely to be found in the last interacted cell (Fact 2), and it is a waste to page the TA (same result is observed for large $V$ with $\lambda_{m} / \lambda_{c}>1$ ). This extra cost becomes significant as the TA 


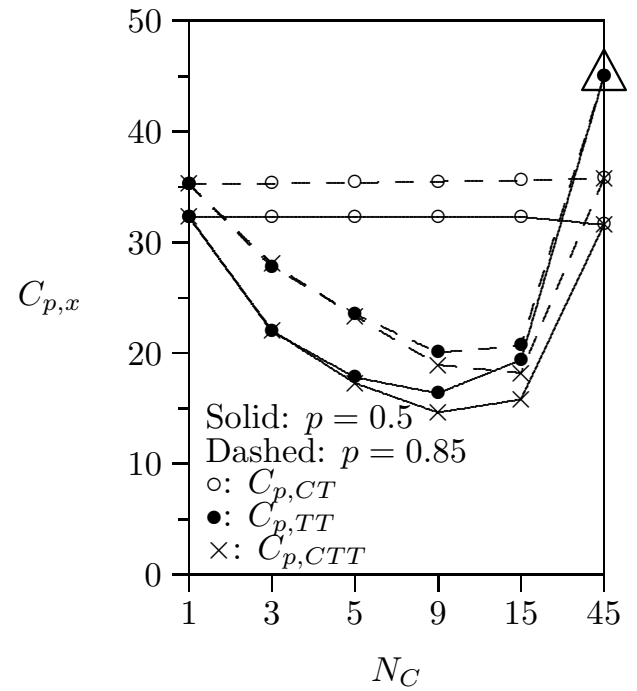

(a) $V=1 / \lambda_{m}^{2}$ and $\lambda_{m} / \lambda_{c}=5$

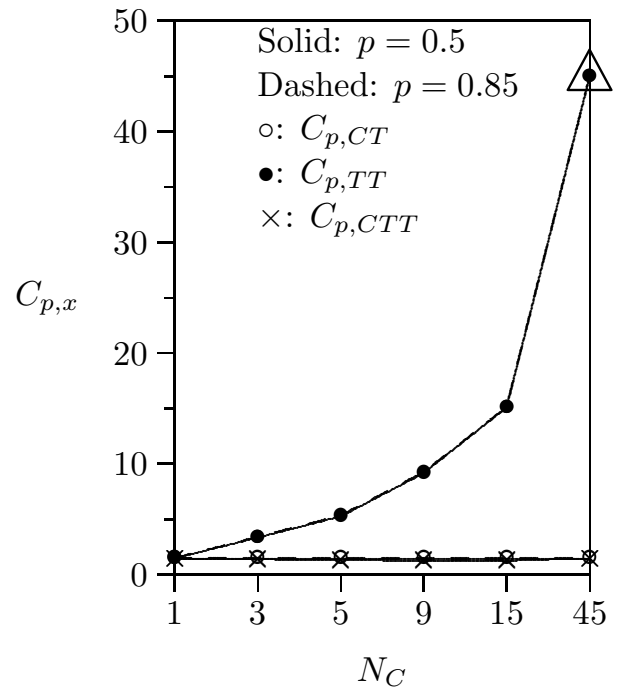

(b) $V=100 / \lambda_{m}^{2}$ and $\lambda_{m} / \lambda_{c}=0.2$

Figure 10: Effects of $N_{C}$ and $p$ on $C_{p, x}\left(N_{C} N_{T}=45\right)$

size is large (i.e., $C_{p, T T}$ increases as $N_{C}$ increases). As we previously stated, the CTT scheme takes advantages of the CT and the TT schemes to yield the best performance for all $N_{C}$ values. This figure also indicates that the lowest $C_{p, x}$ value can be found in the CTT scheme when $5 \leq N_{C} \leq 15$ (or $3 \leq N_{T} \leq 9$ ). Note that when $N_{C}=1$, $C_{p, C T}=C_{p, T T}=C_{p, C T T}$ because a TA only contains one cell and paging the TA is the same as paging the cell (see $(22)$ ). On the other hand, when $N_{C}=45$ (i.e., $N_{T}=1$ ), a TAL only contains one TA, and $C_{p, C T}=C_{p, C T T}$.

As mentioned in Section 2, the $3 \mathrm{G}$ paging is a special case of the TT scheme where $N_{T}=1$ (i.e., $N_{C}=45$; see the $\triangle$ symbol in Figure 10). Figure 10 indicates that the TALbased paging schemes can reduce $21 \%-97 \%$ of the $3 \mathrm{G}$ paging cost. For all input parameter setups under our study, the TAL-based paging schemes always outperform the $3 \mathrm{G}$ paging in terms of the $C_{p, x}$ performance. 


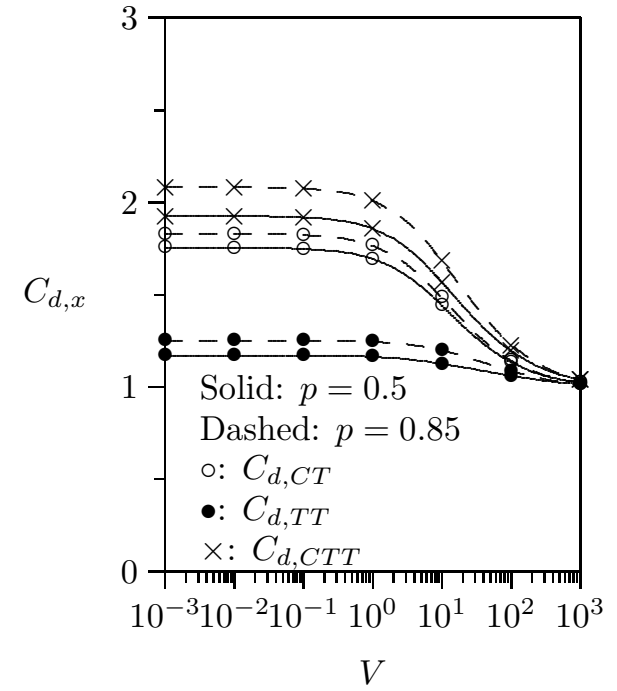

(a) $\lambda_{m} / \lambda_{c}=5$

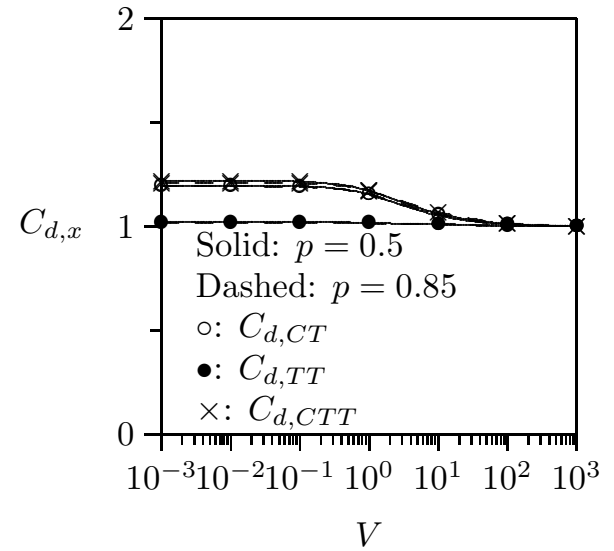

(b) $\lambda_{m} / \lambda_{c}=0.2$

Figure 11: Effects of $\lambda_{m} / \lambda_{c}, p$, and $V$ on $C_{d, x}\left(N_{C}=9\right.$ and $\left.N_{T}=5\right)$

\subsection{Analysis of the $C_{d, x}$ Performance}

Figure 11 plots $C_{d, x}$ against $\lambda_{m} / \lambda_{c}, p$, and $V$. The effects of $\lambda_{m} / \lambda_{c}, p$, and $V$ on $C_{d, x}$ are similar to those on $C_{p, x}$ described in Subsection 4.2, and the details are omitted. It is clear that $C_{d, T T} \leq C_{d, C T} \leq C_{d, C T T}$. As stated in Section 2, the 3G paging simultaneously asks all cells in the LA to page the UE, and thus the polling cycle is 1 . The TAL-based paging schemes incur extra 0.01-1.08 polling cycles over the $3 \mathrm{G}$ paging for high mobility (Figure 11 (a)), and incur extra 0.01-0.21 polling cycles over the 3G paging for low mobility (Figure 11 (b)).

\section{Conclusions}

This paper investigated the performance of LTE mobility management. We considered the central policy for location update and three paging schemes based on the concept of TA and TAL. Our study indicates the following results: 
- The central-based LTE location update outperforms the $3 \mathrm{G}$ location update by $90 \%$ for $p=0.5$ (i.e., when the user movement exhibits locality). On the other hand, the LTE scheme incurs extra 4\%-36\% cost over the $3 \mathrm{G}$ location update for $p=0.85$ (i.e., when the user tends to move to one direction). We note that when $p \leq 0.75$, the TAL-based scheme always outperforms the 3G location update.

- The TAL-based paging schemes outperform the $3 \mathrm{G}$ paging by $21 \%-97 \%$ in terms of the number of paged cells. Among the TAL-based paging schemes, the TT scheme outperforms the CT scheme when the variance $V$ of the cell residence time is small (i.e., the user movement pattern is regular) and the UE moves frequently (i.e., $\lambda_{m} / \lambda_{c}$ is large). The CT scheme outperforms the TT scheme when $V$ is large or $\lambda_{m} / \lambda_{c}$ is small. The CTT scheme takes advantages of both the CT and the TT schemes, which has the best performance in most input parameter setups under our study.

- For the number of polling cycles, the TAL-based paging schemes incur extra 0.01-1.08 polling cycles over the $3 \mathrm{G}$ paging. Among the TAL-based paging schemes, the TT scheme outperforms the CT and the CTT schemes.

In summary, if network signaling costs for location update and paging are major concern, then the CTT scheme should be selected. If the number of polling cycles is major concern, then the existing $3 \mathrm{G}$ mobility management should be selected for the users with high mobility and regular movement patterns (i.e., with small $V$ ). For low mobility users, the extra overheads incurred by the LTE paging can be ignored. Finally, the central-based LTE mobility management creates several interesting research issues. In the future, we will investigate the MME failure restoration based on dynamic TAL assignment [13], power saving [14], mobility management for mesh-mode LTE [15], handoff in heterogeneous network [16], and so on. 


\section{References}

[1] 3GPP. 3rd Generation Partnership Project; Technical Specification Group Services and System Aspects; General Packet Radio Service (GPRS) enhancements for Evolved Universal Terrestrial Radio Access Network (E-UTRAN) access. Technical Specification 3G TS 23.401 version 10.0.0 (2010-06), 2010.

[2] 3GPP. 3rd Generation Partnership Project; Technical Specification Group Radio Access Network; Evolved Universal Terrestrial Radio Access (E-UTRA) and Evolved Universal Terrestrial Radio Access Network (E-UTRAN); Overall description; Stage 2. Technical Specification 3G TS 36.300 version 10.1 .0 (2010-09), 2010.

[3] Amotz Bar-Noy, Ilan Kessler, and Moshe Sidi, "Mobile users: to update or not to update?," Wireless Networks, vol. 1, no. 2, pp. 175-185, 1995.

[4] Joseph S. M. Ho and Ian F. Akyildiz, "Mobile user location update and paging under delay constraints," Wireless Networks, vol. 1, no. 4, pp. 413-425, 1995.

[5] Rong Chen, Senmiao Yuan, and Jinquan Zhu, "A dynamic location management method of personal communication system," E-Tech 2004, pp. 1-9, July 2004.

[6] Ian F. Akyildiz, Joseph S. M. Ho, and Yi-Bing Lin, "Movement-based location update and selective paging for PCS networks," IEEE/ACM Transactions on Networking, vol. 4, no. 4, pp. 629-638, August 1996.

[7] Vincent W.-S. Wong and Victor C. M. Leung, "Location management for nextgeneration personal communications networks," IEEE Network, vol. 14, no. 5, pp. 18-24, September/October 2000.

[8] Ian F. Akyildiz, Janise McNair, Joseph S. M. Ho, Hüseyin Uzunalioğlu, and Wenye Wang, "Mobility management in next-generation wireless systems," Proceedings of the IEEE, vol. 87, no. 8, pp. 1347-1384, August 1999. 
[9] Yi-Bing Lin and Yung-Chun Lin, "WiMAX location update for vehicle applications," Mobile Networks and Applications, vol. 15, no. 1, pp. 148-159, February 2010.

[10] Yi-Bing Lin and Ai-Chun Pang, Wireless and Mobile All-IP Networks. John Wiley \& Sons, Inc., 2005.

[11] Shun-Ren Yang, Yung-Chun Lin, and Yi-Bing Lin, "Performance of mobile telecommunications network with overlapping location area configuration," IEEE Transactions on Vehicular Technology, vol. 57, no. 2, pp. 1285-1292, March 2008.

[12] Frank P. Kelly, Reversibility and Stochastic Networks. New York: Wiley, 1979.

[13] Yi-Bing Lin, "Per-user checkpointing for mobility database failure restoration," IEEE Transactions on Mobile Computing, vol. 4, no. 2, pp. 189-194, March/April 2005.

[14] Shun-Ren Yang, Sheng-Ying Yan, and Hui-Nien Hung, "Modeling UMTS power saving with bursty packet data traffic," IEEE Transactions on Mobile Computing, vol. 6, no. 12, pp. 1398-1409, December 2007.

[15] Di-Wei Huang, Phone Lin, and Chai-Hien Gan, "Design and performance study for a mobility management mechanism (WMM) using location cache for wireless mesh networks," IEEE Transactions on Mobile Computing, vol. 7, no. 5, pp. 546-556, May 2008

[16] Nidal Nasser, Ahmed Hasswa, and Hossam Hassanein, "Handoffs in fourth generation heterogeneous networks," IEEE Communications Magazine, vol. 44, no. 10, pp. 96-103, October 2006. 
Ren-Huang Liou received the B.S. and the M.S. degrees in Computer Science from National Chiao Tung University (NCTU), Hsinchu, Taiwan, R.O.C., in 2007 and 2009, respectively. He is currently working toward the Ph.D. degree at NCTU. His current research interests include Voice over Internet Protocol (VoIP), mobile computing, and performance modeling.

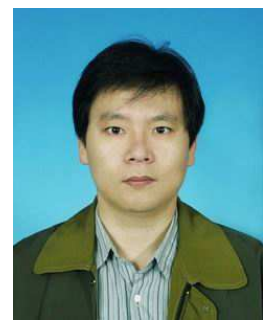

Yi-Bing Lin is Vice President and Life Chair professor of College of Computer Science, National Chiao Tung University (NCTU), and a Visiting professor of King Saud University. He is also with Institute of Information Science and the Research Center for Information Technology Innovation, Academia Sinica, Nankang,Taipei, Taiwan, R.O.C. Lin is the authors of the books Wireless and Mobile Network Architecture (Wiley, 2001), Wireless and Mobile All-IP Networks (John Wiley, 2005), and Charging for Mobile All-IP Telecommunications (Wiley, 2008). Lin received numerous research awards including 2005 NSC Distinguished Researcher and 2006 Academic Award of Ministry of Education. Lin is an ACM Fellow, an AAAS Fellow, an IEEE Fellow and an IET Fellow.

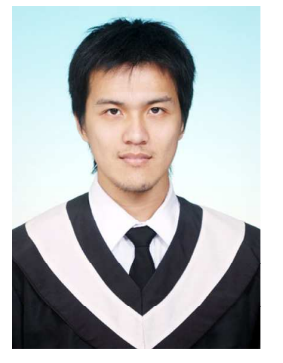

Shang-Chih Tsai received the B.S.C.S. degree from National Chiao Tung University (NCTU), Hsinchu, Taiwan, R.O.C., in 2008. He is currently working toward the M.S.C.S. degree at NCTU. His current research interests include design and analysis of a personal communications services network and performance modeling. 